\title{
Fuzzy Systems with Multiple Rule Bases for Selection of Alternatives using TOPSIS
}

\author{
Abdul Malek Yaakob \\ School of Quantitative Sciences \\ Universiti Utara Malaysia \\ 06010 Sintok Kedah, Malaysia \\ abd.malek@uum.edu.my
}

\author{
Alexander Gegov, Mohammed Bader-El-Den \\ School of Computing \\ University of Portsmouth \\ Buckingham Building, PO1 3HE, United Kingdom \\ \{alexander.gegov, mohamed.bader\}@port.ac.uk
}

\author{
Siti Fatimah Abdul Rahman \\ Department of Mathematics \\ Universiti Teknologi MARA \\ 02600 Arau, Perlis, Malaysia \\ sitifatimah471@uitm.edu.my
}

\begin{abstract}
This paper introduces a novel modification of the technique for ordering of preference by similarity to ideal solution (TOPSIS) method and uses a fuzzy system with multiple rule bases to solve multi-criteria decision making problems where both benefit and cost criteria are presented as subsystems. Thus, the decision maker evaluates the performance of each alternative for optimization and further observes the performance for both benefit and cost criteria. This approach improves significantly the transparency of the TOPSIS method while ensuring high effectiveness in comparison to established methods. To ensure practicality and effectiveness of the proposed method, a traded equity case study is considered. Furthermore, the ranking based on the proposed method is validated comparatively using spearman rho correlation. The proposed method outperforms the existing TOPSIS methods in terms of ranking for the case study under consideration.
\end{abstract}

Keywords-fuzzy systems; multiple rule bases; TOPSIS; multicriteria decision making; spearman rho correlation;Traded equity

\section{INTRODUCTION}

Multi-criteria decision making (MCDM) problems are often observed in reality, and decision makers are faced with the challenge of making decisions in the presence of multiple criteria. The focus is on identifying the best performing solution among feasible alternatives assessed by a group of decision makers and evaluated through multiple criteria [1]. TOPSIS is chosen as the target for the analysis since its stability and simplicity of use with cardinal information [2]. TOPSIS has been successfully applied in MCDM problems as one of the most popular methods used. The main advantage of the TOPSIS method is that it is computationally efficient and easily understood because it directly takes a definitive value from experts to obtain the final result [3].

Fuzzy systems are vital within the armory of fuzzy tools and applicable to real-life decision-making environment. Systems with multiple rule bases are characterised by a white box nature where the inputs are mapped to the outputs through interval variables as connections. According to [4], a multiple rule based fuzzy system is more transparent than a single rule based fuzzy system because it considers separately benefit related and cost related criteria. This system takes into account explicitly the internal structure of the modelled process by representing each group of criteria as a subsystem and the interactions among different groups as connections [5]. This ability brings considerable benefits to modeling complex processes.
Furthermore, existing TOPSIS methods have a very low transparency level and therefore are not able to track the performance of benefit and cost criteria [6]. In decision making processes, it is important that decision makers are aware of how the multiple criteria are performing. Based on [7], it is essential in a decision making environment to track the performance of criteria in order to take control and not underestimate or overestimate the uncertainty of criteria [8]. The proposed method is a systematic TOPSIS approach for estimating the strengths and weaknesses of alternatives that satisfy transactions, activities or functional requirements of organisations. In addition to that, tracking of criteria allows decision makers to determine if it is a sound decision [9]. In this case, it involves comparing the expected cost criteria of each alternative against the expected benefit criteria to see whether the benefits outweigh the costs and by how much. The improvement of the effectiveness described above is the focus of this study.

The paper proposes a novel fuzzy system with multiple rule based modeling method that represents an extension of fuzzy set theory. The method has been validated comparatively against established fuzzy system based modelling methods for a case study on ranking of equities. The main advantages of the proposed method in the context of this case study are its higher transparency and accuracy. The paper is structured as follows: Section II briefly reviews the basic concepts of fuzzy sets. The novel methodology of TOPSIS using fuzzy systems with multiple rule bases (MFS-TOPSIS) is formulated in Section III. Section IV illustrates the applicability of the proposed method to the problem of ranking traded equities. Further discussion and analysis of the proposed method ranking performance are provided in Section V. The main conclusions are summarised in Section VI.

\section{BASIC CONCEPT}

In the following, some basic definitions of fuzzy sets from [10], [11] is briefly review. These basic definitions and notations are used throughout the paper unless stated otherwise.

Definition 1[12] : Fuzzy set

A fuzzy set $A$ is defined on a universe $X$ may be given as: 
$A=\left\{\left(x, \mu_{A_{i}}(x)\right) \mid x \in X\right\}, \quad$ where $\quad \mu_{A_{i}}(x): X \rightarrow[0,1] \quad$ is the membership function of $A$. The membership value $\mu_{A_{i}}(x)$ describes the degree of belongingness of $x \in X$ in $A$.

Throughout this paper, fuzzy number is presented in the form of trapezoidal fuzzy number. It is easy to deal with because it is piece wise linear. On the other hand, the good coverage of trapezoidal fuzzy number is a good compromise between efficiency and effectiveness.

Definition 2 [12]: Fuzzy Number

A trapezoidal fuzzy numbers can be represented by the following membership function given by

$$
\mu_{A_{i}}(x)=\left(a_{i 1}, a_{i 2}, a_{i 3}, a_{i 4}\right)=\left\{\begin{array}{ccc}
\frac{x-a_{i 1}}{a_{i 2}-a_{i 1}} & \text { if } & a_{i 1} \leq x \leq a_{i 2} \\
1 & \text { if } & a_{i 2} \leq x \leq a_{i 3} \\
\frac{a_{i 4}-x}{a_{i 4}-a_{i 3}} & \text { if } & a_{i 3} \leq x \leq a_{i 4} \\
0 & & \text { otherwise }
\end{array}\right.
$$

\section{PROPOSED METHODS}

In this section, the TOPSIS with single rule bases proposed [13] is extended using multiple rule bases approach. The main aim of the extension is to apply the ability of multiple rule bases in to decision process, in particular TOPSIS analysis, which is to improve the level of transparency for each criteria. In this approach, the criteria are divided into two categories, called benefit rule base and cost rule base with benefit inputs ( $\left.B_{e}\right)$, and cost inputs $\left(C_{f}\right)$. Outputs of the rule bases namely benefit level (BL) and cost level (CL) respectively. By this way, the decision maker can trace the performance of benefit criteria and cost criteria.

Table 1-2 represent detail the linguistic terms for the importance of weight of each criterion and rating of the alternative respectively.

TABLE 1: LINGUISTIC TERMS FOR IMPORTANCE OF WEIGHT OF EACH CRITERION

\begin{tabular}{|c|c|}
\hline Linguistic Terms & Trapezoidal Fuzzy Number \\
\hline Very low (VL) & $\left(\begin{array}{llll}0.00 & 0.00 & 0.00 & 0.10\end{array}\right)$ \\
\hline Low (L) & $\left(\begin{array}{llll}0.00 & 0.10 & 0.10 & 0.25\end{array}\right)$ \\
\hline Medium low (ML) & $\left(\begin{array}{llll}0.15 & 0.30 & 0.30 & 0.45\end{array}\right)$ \\
\hline Medium $(\mathrm{M})$ & $\left(\begin{array}{llll}0.35 & 0.50 & 0.50 & 0.65\end{array}\right)$ \\
\hline Medium high (MH) & $\left(\begin{array}{llll}0.55 & 0.70 & 0.70 & 0.85\end{array}\right)$ \\
\hline $\operatorname{High}(\mathrm{H})$ & $\left(\begin{array}{llll}0.80 & 0.90 & 0.90 & 1.00\end{array}\right)$ \\
\hline Very high (VH) & $\left(\begin{array}{llll}0.90 & 1.00 & 1.00 & 1.00\end{array}\right)$ \\
\hline
\end{tabular}

TABLE 2: LINGUISTIC TERMS FOR RATING OF ALL ALTERNATIVE

\begin{tabular}{|c|c|}
\hline Linguistic Terms & Trapezoidal Fuzzy Number \\
\hline Very poor (VP) & $\left(\begin{array}{lllll}0 & 0 & 0 & 1\end{array}\right)$ \\
\hline Poor (P) & $\left(\begin{array}{lllll}0 & 1 & 1 & 3\end{array}\right)$ \\
\hline Medium poor (MP) & $\left(\begin{array}{llll}1 & 3 & 3 & 5\end{array}\right)$ \\
\hline Fair $(\mathrm{F})$ & $\left(\begin{array}{llll}3 & 5 & 5 & 7\end{array}\right)$ \\
\hline Medium good (MG) & $\left(\begin{array}{llll}5 & 7 & 7 & 9\end{array}\right)$ \\
\hline Good (G) & $\left(\begin{array}{llll}7 & 9 & 9 & 10\end{array}\right)$ \\
\hline Very good (VG) & $\left(\begin{array}{llll}9 & 10 & 10 & 10\end{array}\right)$ \\
\hline
\end{tabular}

The linguistic terms as shown in Table 3, which represents the consequents of rules was named "Alternative Level" and is represented by fuzzy sets "Very bad", "Bad", "Regular", "Good" and "Very Good".

TABLE 3: LINGUISTIC VARIABLE FOR ALTERNATIVE LEVEL

\begin{tabular}{|l|c|}
\hline Linguistic Terms & Trapezoidal Fuzzy Number \\
\hline Very Bad (VB) & $(0.000 .000 .000 .25)$ \\
\hline Bad (B) & $(0.000 .250 .250 .50)$ \\
\hline Regular (R) & $(0.250 .500 .500 .75)$ \\
\hline Good (G) & $(0.500 .750 .751 .00)$ \\
\hline Very good $(\mathrm{VG})$ & $(0.751 .001 .001 .00)$ \\
\hline
\end{tabular}

The following algorithm is conducted to obtain the ranking of alternatives, whereby Step 1-5 are adopted from [10]. In order to deal with influence degree of decision maker step 6-11 have introduced in this paper, which make use the rule based approach.

\section{MFS-TOPSIS Formulation}

Step 1: Construct decision matrix where each decision maker opinion is evaluated independently and categorise into two Criteria Categories as Benefit Criteria and Cost Criteria define through a Benefit system (BS) and a Cost system (CS))

In the decision matrices $D_{k}^{B}, D_{k}^{C}$ and weight matrices $W_{k}^{B}, W_{k}^{C}(k=1, \cdots, K)$, it is assumed that $e$ is the number of benefit criteria and $f$ is the number of cost criteria, as shown in Eq. (1):

$$
\begin{aligned}
& D_{k}^{B}=\begin{array}{c}
B_{1} \\
B_{2} \\
\vdots \\
B_{e}
\end{array}\left[\begin{array}{cccc}
x_{11, k} & x_{12, k} & \cdots & x_{1 m, k} \\
x_{21, k} & x_{22, k} & \cdots & x_{2 m, k} \\
\vdots & \vdots & \ddots & \vdots \\
x_{e 1, k} & x_{e 2, k} & \cdots & x_{e m, k}
\end{array}\right] \text { and } \\
& D_{k}^{C}=\begin{array}{c}
C_{1} \\
C_{2} \\
\vdots \\
C_{f}
\end{array}\left[\begin{array}{cccc}
y_{11, k} & y_{12, k} & \cdots & y_{1 m, k} \\
y_{21, k} & y_{22, k} & \cdots & y_{2 m, k} \\
\vdots & \vdots & \ddots & \vdots \\
y_{f 1, k} & y_{f 2, k} & \cdots & y_{f m, k}
\end{array}\right] ; \\
& W_{k}^{B}=\left[\begin{array}{llll}
g_{1, k} & g_{2, k} & \cdots & g_{e, k}
\end{array}\right] \text { and } \\
& W_{k}^{C}=\left[\begin{array}{llll}
h_{1, k} & h_{2, k} & \cdots & h_{f, k}
\end{array}\right] \text {, for } k=1, \cdots, K \text {. }
\end{aligned}
$$

where $x_{i j, k}$ are fuzzy sets representing the rating of alternatives $A_{j}(j=1, \cdots, m)$ with respect to benefit criteria $B_{i}(i=1, \cdots, e)$ according to the $k^{\text {th }}$ decision maker, and $g_{i, k}$ are fuzzy sets representing the weights of benefit criteria $B_{i, k}$ $(i=1, \cdots, e)$ according to the $k^{\text {th }}$ decision maker, where $k=1, \cdots, K$. Also, $y_{i j, k}$ are fuzzy sets describing the rating of alternatives $A_{j}(j=1, \cdots, m)$ with respect to cost criteria $C_{i}$ $(i=1, \cdots, f)$ according to the $k^{\text {th }}$ decision maker, and $h_{i, k}$ are fuzzy sets describing the weights of cost criteria $C_{i}$ 
$(i=1, \cdots, f)$ according to the $k^{t h}$ decision maker, where $k=1, \cdots, K$.

Step 2: Construct weighted and normalized decision matrices

The fuzzy rating and weight of each criterion are terms described with trapezoidal fuzzy numbers. The ratings of alternatives $A_{j}(j=1, \cdots, m)$ are described with the trapezoidal fuzzy numbers $x_{i j, k}=\left(\begin{array}{llll}a_{i j, k}^{x} & b_{i j, k}^{x} & c_{i j, k}^{x} & d_{i j, k}^{x}\end{array}\right)$ and $y_{i j, k}=\left(\begin{array}{llll}a_{i j, k}^{y} & b_{i j, k}^{y} & c_{i j, k}^{y} & d_{i j, k}^{y}\end{array}\right)$, while the importance of benefit criteria $B_{i}(i=1, \cdots, e)$ and cost criteria $C_{i}(i=1, \cdots, f)$ are respectively represented by $g_{i, k}=\left(\begin{array}{llll}a_{i, k}^{g} & b_{i, k}^{g} & c_{i, k}^{g} & d_{i, k}^{g}\end{array}\right)$ and $h_{i, k}=\left(\begin{array}{llll}a_{i, k}^{h} & b_{i, k}^{h} & c_{i, k}^{h} & d_{i, k}^{h}\end{array}\right)$, for $k=1, \cdots, K$. The normalized fuzzy decision matrices $R_{k}$ and weight normalized fuzzy decision matrices $V_{k}$ are calculated as shown in Eq. (2):

$$
R_{k}=\left[r_{i j, k}\right]_{(e+f) \times m}
$$

Where

$$
\begin{aligned}
& r_{i j, k}=\left\{\begin{array}{l}
r_{i j . k}^{B}=\left(\frac{a_{i j, k}^{x}}{d_{i, k}^{x^{*}}}, \frac{b_{i j, k}^{x}}{d_{i, k}^{x^{*}}}, \frac{c_{i j, k}^{x}}{d_{i, k}^{x^{*}}}, \frac{d_{i j, k}^{x}}{d_{i, k}^{x^{*}}}\right), \text { for } B_{i} \in B \\
r_{i j . k}^{C}=\left(\frac{a_{i, k}^{y^{*}}}{d_{i j, k}^{y}}, \frac{a_{i, k}^{y^{*}}}{c_{i j, k}^{y}}, \frac{a_{i, k}^{y^{*}}}{b_{i j, k}^{y}}, \frac{a_{i, k}^{y^{*}}}{a_{i j, k}^{y}}\right), \text { for } C_{i} \in C
\end{array}\right. \\
& d_{i, k}^{x^{*}}=\max _{j} d_{i j, k}^{x},(i=1, \cdots, e),(j=1, \cdots, m) \\
& a_{i, k}^{y^{*}}=\min _{j} a_{i j, k}^{y},(i=1, \cdots, f),(j=1, \cdots, m)
\end{aligned}
$$

$B$ and $C$ are the sets of benefit criteria and cost criteria respectively;

$$
\begin{array}{ll}
V_{k}=\left[v_{i j, k}\right]_{(e+f) \times m}, \text { where } & \\
v_{i j, k}= \begin{cases}v_{i j . k}^{B}=r_{i j, k}(\cdot) g_{i, k} & , \text { for } B_{i} \in B \\
v_{i j . k}^{C}=r_{i j, k}(\cdot) h_{i, k} & , \text { for } C_{i} \in C\end{cases}
\end{array}
$$

And $v_{i j, k}=\left(\begin{array}{llll}a_{i j, k}^{v} & b_{i j, k}^{v} & c_{i j, k}^{v} & d_{i j, k}^{v}\end{array}\right)$ are fuzzy sets;

for $k=1, \cdots, K$.

Step 3: Find the Fuzzy Positive Ideal Solution (FPIS) and Fuzzy Negative Ideal Solution (FNIS) for each alternative.

The FPIS and FNIS solutions are correspondingly $A_{k}^{+}=\left(v_{1, k}^{+}, v_{2, k}^{+}, \cdots, v_{(e+f), k}^{+}\right)$and $A_{k}^{-}=\left(v_{1, k}^{-}, v_{2, k}^{-}, \cdots, v_{(e+f), k}^{-}\right)$, where $v_{i j, k}^{+}=\left(\begin{array}{llll}1 & 1 & 1 & 1\end{array}\right)$ and $v_{i j}^{-}=\left(\begin{array}{llll}0 & 0 & 0 & 0\end{array}\right)$ are fuzzy sets, for $k=1, \cdots, K$.

Step 4: Find the distance between each alternative to FPIS and FNIS.

The distance for benefit criteria of each alternative $j$ from $A_{k}^{+}$is $\Delta_{j, k}^{B+}$, calculated as shown in Eq. (3):

$$
\begin{aligned}
& \Delta_{j, k}^{B+}=\sum_{i=1}^{e} \Delta_{k}^{B}\left(v_{i j, k}^{B}, v_{i, k}^{+}\right), \text {where } \\
& \Delta_{k}^{B}\left(v_{i j, k}^{B}, v_{i, k}^{+}\right) \\
& =\sqrt{\frac{1}{3}\left[\begin{array}{l}
\left(a_{i j, k}^{v, B}-1\right)^{2}+\left(b_{i j, k}^{v, B}-1\right)^{2} \\
+\left(c_{i j, k}^{v, B}-1\right)^{2}+\left(d_{i j, k}^{v, B}-1\right)^{2}
\end{array}\right]} \text { for } \\
& j=1, \cdots, m \text {, and } B_{i} \in B \text {, and } k=1, \cdots, K \text {. }
\end{aligned}
$$

The distance for benefit criteria of each alternative from $A_{j, k}^{-}$is $\Delta_{j, k}^{B-}$, calculated as shown in Eq. (4):

$$
\Delta_{j, k}^{B-}=\sum_{i=1}^{e} \Delta_{k}^{B}\left(v_{i j, k}^{B}, v_{i, k}^{-}\right)
$$

where

$$
\Delta_{k}^{B}\left(v_{i j, k}^{B}, v_{i, k}^{-}\right)
$$

$$
\begin{gathered}
=\sqrt{\frac{1}{3}\left[\begin{array}{l}
\left(a_{i j, k}^{v, B}-0\right)^{2}+\left(b_{i j, k}^{v, B}-0\right)^{2} \\
+\left(c_{i j, k}^{v, B}-0\right)^{2}+\left(d_{i j, k}^{v, B}-0\right)^{2}
\end{array}\right]} \text { for } \\
j=1, \cdots, m \text {, and } B_{i} \in B \text {, and } k=1, \cdots, K
\end{gathered}
$$

The distance for cost criteria of each alternative from $A_{k}^{+}$is $\Delta_{j, k}^{C+}$, calculated as shown in Eq. (5):

$$
\Delta_{j, k}^{C+}=\sum_{i=1}^{f} \Delta_{k}^{C}\left(v_{i j, k}^{C}, v_{i, k}^{+}\right),
$$

where

$$
\begin{aligned}
& \Delta_{k}^{C}\left(v_{i j, k}^{C}, v_{i, k}^{+}\right) \\
& =\sqrt{\frac{1}{3}\left[\begin{array}{l}
\left.\left(a_{i j, k}^{v, C}-1\right)^{2}+\left(b_{i j, k}^{v, C}-1\right)^{2}\right] \\
+\left(c_{i j, k}^{v, C}-1\right)^{2}+\left(d_{i j, k}^{v, C}-1\right)^{2}
\end{array}\right]}, \\
& \text { for } j=1, \cdots, m \quad, \text { and } C_{i} \in C, \text { and }
\end{aligned}
$$$$
k=1, \cdots, K \text {. }
$$

Finally, the distance for cost criteria of each alternative from

$A_{k}^{-}$is $\Delta_{j, k}^{C-}$, calculated as shown in Eq. (6):

$$
\Delta_{j, k}^{C-}=\sum_{i=1}^{f} \Delta_{k}^{C}\left(v_{i j, k}^{C}, v_{i, k}^{-}\right),
$$

where

$$
\begin{aligned}
& \Delta_{k}^{C}\left(v_{i j, k}^{C}, v_{i, k}^{-}\right) \\
& =\sqrt{\frac{1}{3}\left[\begin{array}{l}
\left(a_{i j, k}^{v, C}-0\right)^{2}+\left(b_{i j, k}^{v, C}-0\right)^{2} \\
+\left(c_{i j, k}^{v, C}-0\right)^{2}+\left(d_{i j, k}^{v, C}-0\right)^{2}
\end{array}\right]}
\end{aligned}
$$$$
\text { for } j=1, \cdots, m \text {, and } C_{i} \in C \text {, and } k=1, \cdots, K
$$ 
Step 5: Find the Closeness Coefficients (CC) for the benefit and cost systems.

The closeness coefficients $C C_{j, k}^{B}$ for the benefit systems, and the closeness coefficients $C C_{j, k}^{C}$ for the cost systems, are calculated in Eq. (7):

$$
\begin{gathered}
C C_{j, k}^{B}=\frac{\Delta_{j, k}^{B-}}{\Delta_{j, k}^{B+}+\Delta_{j, k}^{B-}} \quad \text { and } \\
C C_{j, k}^{C}=\frac{\Delta_{j, k}^{C-}}{\Delta_{j, k}^{C+}+\Delta_{j, k}^{C-}} \text { for } j=1, \cdots, m \text { and } \\
k=1, \cdots, K .
\end{gathered}
$$

Step 6: Derive the Influenced Closeness Coefficient (ICC) by applying the influence degree of each decision maker, then find Normalised ICC (NICC), dividing NICC by maximum value of NICC.

Let $\theta_{k}$ denotes the influence degree, between 0 (uninfluential) and 10 (very influential), of decision maker $k$, where $k=1, \cdots, K$. Next, let $\sigma_{k}$ stands for the normalized influence degree of the $k^{\text {th }}$ decision maker, $k=1, \cdots, K$, as evaluated with Eq. (8):

$$
\sigma_{k}=\theta_{k} / \sum_{l=1}^{K} \theta_{l} \quad, \text { for } k=1, \cdots, K .
$$

Eq. (9) evaluates the influence closeness coefficients $I C C_{j, k}^{B}$ and $I C C_{j, k}^{C}$ for each DM $k$, respectively along the benefit and cost criteria.

$$
\begin{aligned}
I C C_{j, k}^{B} & =\sigma_{k} * C C_{j, k}^{B} \text { and } \\
I C C_{j, k}^{C} & =\sigma_{k} * C C_{j, k}^{C}
\end{aligned}
$$

for $j=1, \cdots, m$ and $k=1, \cdots, K$.

It is further necessary to normalize the coefficients, in order to ensure that their values vary between 0 to 1 . Eq. (10) evaluates the normalised coefficients, where $N I C C_{j, k}^{B}$ and $N I C C_{j, k}^{C}$ are respectively the normalized influence closeness coefficients for the benefit and cost systems, as related to the $k^{\text {th }}$ decision maker.

$$
\begin{array}{ll}
N I C C_{j, k}^{B}=I C C_{j, k}^{B} / \max _{j} I C C_{j, k}^{B} & \text { and } \\
N I C C_{j, k}^{C}=I C C_{j, k}^{C} / \max _{j} I C C_{j, k}^{C} \text { for } j=1, \cdots, m & \text { and } \\
k=1, \cdots, K . &
\end{array}
$$

Both $N I C C_{j, k}^{B}$ and $N I C C_{j, k}^{C}$ will take linguistic terms from Table 3 for the level of alternatives performance.

Step 7: Construct the antecedent matrices for the BS and CS based on DMs opinions

Having the opinions $D_{k}^{B}$ and $D_{k}^{C}$ of all DMs $(k=1, \cdots, K)$ on each alternative $j(j=1, \cdots, m)$ in respect to each benefit criterion $i(i=1, \cdots, e)$ and each cost criterion $i(i=1, \cdots, f)$, we can define the BS antecedent matrix $X_{k}$ and the CS antecedent matrix $Y_{k}$ for each DM $k$, as introduced with Eq. (11):

$$
\begin{aligned}
X_{k} & =\left[\begin{array}{cccc}
x_{11, k} & x_{12, k} & \cdots & x_{1 m, k} \\
x_{21, k} & x_{22}, & \cdots & x_{2 m, k} \\
\vdots & \vdots & \ddots & \vdots \\
x_{e 1, k} & x_{e 2, k} & \cdots & x_{e m, k}
\end{array}\right] \\
Y_{k} & =\left[\begin{array}{cccc}
y_{11, k} & y_{12, k} & \cdots & y_{1 m, k} \\
y_{21, k} & y_{22, k} & \cdots & y_{2 m, k} \\
\vdots & \vdots & \ddots & \vdots \\
y_{f 1, k} & y_{f 2, k} & \cdots & y_{f m, k}
\end{array}\right] \text { for } k=1, \cdots, K,
\end{aligned}
$$

describing decision makers' opinions.

Step 8: Construct the consequent matrices for the BS and $C S$ systems based on the value of the NICC coefficients.

Having determined the $N I C C_{j}^{B, k}$ and $N I C C_{j}^{C, k}$ coefficients for all decision makers $(k=1, \cdots, K)$, next the benefit consequent matrix $\Lambda_{k}$ and the cost consequent matrix $\Psi_{k}$ are defined as shown in Eq. (12):

$$
\begin{aligned}
\Lambda_{k} & =\left[\begin{array}{llll}
\lambda_{1, k} & \lambda_{2, k} & \cdots & \lambda_{m, k}
\end{array}\right] \text { and } \\
\Psi_{k} & =\left[\begin{array}{llll}
\psi_{1, k} & \psi_{2, k} & \cdots & \psi_{m, k}
\end{array}\right] \\
& \text { for } k=1, \cdots, K,
\end{aligned}
$$

where $\lambda_{i, k}$ and $\psi_{i, k}$ are linguistic terms representing the output of the BS and CS systems, based respectively on the values of $N I C C_{j, k}^{B}$ and $N I C C_{j, k}^{C}$.

Step 9: Derive rules for each alternative for benefit and cost subsystem

The rule base of benefit subsystem for DM1 is constructed using Eq. (11) and Eq. (12), as in Eq. (13):

$$
\text { If } \quad X_{k}=\left[\begin{array}{cccc}
x_{11, k} & x_{12, k} & \cdots & x_{1 m, k} \\
x_{21, k} & x_{22}, & \cdots & x_{2 m, k} \\
\vdots & \vdots & \ddots & \vdots \\
x_{e 1, k} & x_{e 2, k} & \cdots & x_{e m, k}
\end{array}\right]
$$

Then $\quad \Lambda_{k}=\left[\begin{array}{llll}\lambda_{1, k} & \lambda_{2, k} & \cdots & \lambda_{m, k}\end{array}\right]$;

Rule 1: If $B_{1}$ is $x_{1 j, 1}$ and $\cdots$ and $B_{e}$ is $x_{e j, 1}$ and $C_{1}$ is $y_{1 j, 1}$ and $\cdots$ and $C_{f}$ is $y_{f j, 1}$ then $A L$ is $N \xi_{j, 1}$

Rule $n_{j}$ : If $B_{1}$ is $x_{1 j, K}$ and $\cdots$ and $B_{e}$ is $x_{e j, K}$ and $C_{1}$ is $y_{1 j, K}$ and $\cdots$ and $C_{f}$ is $y_{f j, K}$ then $A L$ is $N \xi_{j, K}$

By analogy, the rule bases for cost subsystem constructed

Step 10: Derive the weighted benefit level (WBL) and weighted cost level (WCL) 
The weighted benefit level and weighted cost level are derive by taking average of the aggregate membership value of consequent part of all active rules multiply with weight of system based on number of input for each system. And then multiplying with the influence multiplier [14] based on the average as shown in Eq.(14)

$$
W B L_{j, k}=\left[\frac{1}{n} \sum_{\text {rule }=1}^{n} \hat{\mu}_{j, k}^{B} \times\left(\frac{e}{e+f}\right)\right] \times \frac{1}{k} \sum_{k=1}^{k} N I C C_{j, k}^{B}
$$

and for cost system

$$
W C L_{j, k}=\left[\frac{1}{n} \sum_{\text {rule }=1}^{n} \hat{\mu}_{j, k}^{C} \times\left(\frac{f}{e+f}\right)\right] \times \frac{1}{k} \sum_{k=1}^{k} N I C C_{j, k}^{C}
$$

Step 11: Derive final score for each alternative,

In order to produce a final score $\Gamma_{j}$ for each alternative $j$, take the average aggregate membership value of the consequent part of the $n_{j}$ rules in Eq. (13). Then multiply with the influence multiplier based on the $K$ DMs average influence degree for alternative $j$. This is shown in Eq. (15):

$$
\Gamma_{j}=\left(W B L_{j, k}+W C L_{j, k}\right) / 2 \quad \text {, for } j=1, \cdots, m .
$$

Step 12: Finally rank alternative base on final score value, the higher final value the better the alternative performance.

Thus the ranking order of all alternatives can be determined: the better alternatives $j$ have higher values of $\Gamma_{j}$.

\section{CASE STUDY}

We study the problem of ranking equity in developing financial markets within a crisis period, in order to illustrate the applicability and validity of the proposed methodology in a realistic scenario. Decision makers with different levels of experience evaluate 25 equities listed on the Main Board of the Kuala Lumpur Stock Exchange (KLSE) on 30 November 2007.

A set of financial ratios for the equities are considered towards the benefits and cost criteria in the multiple rule bases algorithm. These include Market Value of Firm (B1), defined as market value of firm-to-earnings before amortization, interest and taxes [15]; Return on Equity (B2), which evaluates how much the company earns on the investment of its shareholders. The higher values of the ratio indicate healthier companies. Debt-to-Equity ratio (C1), belonging to long-term solvency ratios that are intended to address the firm's long run ability to meet its obligations. It is considered by DMs that the lower the ratio the better [16]. Current Ratio (B3), which measures liquidity of companies. The higher the ratio, the more liquid is the company, and therefore in a better position [21]. Market Value-to-Net Sales (B4), is market value ratios of particular interest to investors. The lower the ratio the better the equity [17]. The lower this ratio is better the equity. Price/earnings ratio (C2), measure the ratio of market price of each share of common equity to the earnings per share, the lower this ratio is better.

In this study, the processes of ranking equities as discussed in section III which consider fuzzy system using multiple rule bases to solve equity selection. Figure 2 illustrates the proposed method for the problem and the criteria considered are 4 benefit criteria and 2 cost criteria.
Step 1: Based on the information provided by experts and using Eq. (1), the decision matrices for the benefit and cost systems can be constructed. The linguistic terms in Tables 4, -7 can be converted by using the fuzzy numbers in Tables 1 and 2 respectively. The rating of each criterion for each equity and the importance of criteria are based on decision makers opinions.

Step 2: Considering the benefit system, the normalized decision matrix $R_{k}^{B}$ and the weight normalized decision matrix $V_{k}^{B}$ can be constructed for each $k$, using equations Eq. (2) correspondingly.

For example, the calculations for E1 using the opinion of DM1 is as follows:

$$
\begin{gathered}
g_{1,1}=(0.9,1,1,1) x_{11,1}=(9,10,10,10) d_{1,1}^{x^{*}}=10 \\
r_{11,1}^{B}=(9 / 10,10 / 10,10 / 10,10 / 10)=(0.9,1,1,1) \\
v_{11,1}^{B}=(0.9 \times 0.9,1 \times 1,1 \times 1,1 \times 1)=(0.81,1,1,1)
\end{gathered}
$$

This step is repeated then for the cost system, in order to calculate the normalized decision matrix $R_{k}^{C}$ and the weight normalized decision matrix $V_{k}^{C}$.

Step 3: The Fuzzy Positive Ideal Solution (FPIS) and the Fuzzy Negative Ideal Solution (FNIS) for each equity based on both systems, and the distances between the rating of criteria for each equity and the FPIS and FNIS, can be evaluated as follows.

FPIS and FNIS are determined as:

$$
\begin{aligned}
& A_{k}^{+}=\left[(1,1,1,1)_{1, k},(1,1,1,1)_{2, k}, \ldots,(1,1,1,1)_{25, k}\right] \\
& A_{k}^{-}=\left[(0,0,0,0)_{1, k},(0,0,0,0)_{2, k}, \ldots,(0,0,0,0)_{25, k}\right]
\end{aligned}
$$

Step 4: The distances $\Delta_{j, k}^{B+}$ and $\Delta_{j, k}^{B+}$, between the rating according to DM $\mathrm{k}$ of benefit criteria $i=1, \ldots, 4$ for each equity $j(j=1, \ldots, 25)$ and the FPIS $A_{k}^{+}$or FNIS $A_{k}^{-}$are calculated using Eq. (3) and Eq. (4). For example, the distance between the first equity E1 according to DM1and the FPIS $A_{1}^{+}$is calculated using Eq. (3) for $j=1$ and $k=1$, as follows:

$$
\begin{aligned}
& \Delta_{k}^{B+}\left(v_{i j, k}, v_{i, k}^{+}\right)=\Delta_{1}^{B+}\left(v_{11,1}, v_{1,1}^{+}\right) \\
& =\sqrt{\frac{1}{3}\left[(0.81-1)^{2}+\cdots+(1-1)^{2}\right]}=0.11
\end{aligned}
$$

And similarly:

$$
\begin{aligned}
& \Delta_{1}^{B+}\left(v_{21,1}, v_{2,1}^{+}\right)=0.409 ; \Delta_{1}^{B+}\left(v_{31,1}, v_{3,1}^{+}\right)=0.668 \\
& \Delta_{1}^{B+}\left(v_{31,1}, v_{3,1}^{+}\right)=0.298
\end{aligned}
$$

to produce overall:

$$
\Delta_{j, k}^{B+}=0.11+0.409+0.668+0.298=1.4841
$$

Next, using Eq. (4) for $j=1$ and $k=1$, the distance between E1 according to DM1 and the FPIS $A_{1}^{-}$is calculated as:

$$
\Delta_{k}^{B-}\left(v_{i j, k}, v_{i, k}^{-}\right)=\sqrt{\frac{1}{3}\left[(0.81-0)^{2}+\cdots+(1-0)^{2}\right]}=1.373
$$

and similarly 
$\Delta_{1}^{B-}\left(v_{21,1}, v_{2,1}^{-}\right)=1.063 ; \Delta_{1}^{B-}\left(v_{31,1}, v_{3,1}^{-}\right)=0.789$

$\Delta_{1}^{B-}\left(v_{31,1}, v_{3,1}^{-}\right)=1.242$

producing overall:

$\Delta_{1,1}^{B-}=1.373+1.063+0.789+1.242=4.4671$

Now, the distances $\Delta_{j, k}^{C+}$ and $\Delta_{j, k}^{C-}$, between the rating according to DM $\mathrm{k}$ of cost criteria $i=1, \ldots, 2$ for each equity $j$ $(j=1, \ldots, 25)$ and the FPIS $A_{k}^{+}$or FNIS $A_{k}^{-}$are calculated using Eq. (5) and Eq. (6). For example, the distance between the first equity E1 according to DM1 and the FPIS $A_{1}^{+}$is calculated using Eq. (3) for $j=1$ and $k=1$, as follows:

$$
\Delta_{1}^{C+}\left(v_{11,1}, v_{1,1}^{+}\right)=\sqrt{\frac{1}{3}\left[(0.39-1)^{2}+\cdots+(0.85-1)^{2}\right]}=0.49
$$

And similarly:

$\Delta_{k}^{C+}\left(v_{i j, k}, v_{i, k}^{+}\right)=\Delta_{1}^{C+}\left(v_{21,1}, v_{2,1}^{+}\right)=1.12$

to produce overall:

$$
\Delta_{j, k}^{C+}=\sum_{i=1}^{i} \Delta_{k}^{C}\left(v_{1 j, k}, v_{1, k}^{+}\right)=\Delta_{1,1}^{C+}=\sum_{i=1}^{2} \Delta_{1}^{C}\left(v_{i 1,1}, v_{i, 1}^{+}\right)
$$

$=0.49+1.12=1.61$

Next, using Eq. (6) for $j=1$ and $k=1$, the distance between E1 according to DM1 and the FPIS $A_{1}^{-}$is calculated as:

$$
\Delta_{1}^{C-}\left(v_{11,1}, v_{1,1}^{-}\right)=\sqrt{\frac{1}{3}\left[(0.39-0)^{2}+\cdots+(0.85-0)^{2}\right]}=1.017
$$

and similarly

$$
\Delta_{k}^{C-}\left(v_{i j, k}, v_{i, k}^{-}\right)=\Delta_{1}^{C-}\left(v_{21,1}, v_{2,1}^{-}\right)=0.339
$$

producing overall:

$$
\Delta_{j, k}^{C-}=\sum_{i=1}^{i} \Delta_{k}^{C-}\left(v_{1 j, k}, v_{1, k}^{-}\right)=\Delta_{1,1}^{C-}=\sum_{i=1}^{2} \Delta_{1}^{C-}\left(v_{i 1,1}, v_{i, 1}^{-}\right)
$$

$=1.017+0.339=1.358$

Step 5: Find the closeness coefficients for the benefit system $C C_{j, k}^{B}$ and for the cost system $C C_{j, k}^{C}$, using Eq. (7) for each equity $\mathrm{E} j, j=1, \ldots, 25$. For example, the closeness coefficient for E1 in the benefit system under the first decision maker $k=1$ is calculated using Eq. (7) as follows:

$$
C C_{1,1}^{B}=\frac{\Delta_{1,1}^{B-}}{\Delta_{1,1}^{B+}+\Delta_{1,1}^{B-}}=\frac{4.4671}{1.4841+4.4671}=0.751
$$

and the closeness coefficient in the cost system

$$
C C_{1,1}^{C}=\frac{\Delta_{1,1}^{C-}}{\Delta_{1,1}^{C+}+\Delta_{1,1}^{C-}}=\frac{1.358}{1.61+1.358}=0.457
$$

Step 6: The Influenced Closeness Coefficients $I C C_{j, k}^{B}$ and $I C C_{j, k}^{C}$ for each DM $k$ are derived by applying the influence degree $\theta_{k}$ of each decision maker, Using Eq. (8) and Eq. (9).
Then the normalized coefficients $N I C C_{j, k}^{B}$ and $N I C C_{j, k}^{C}$ are calculated with Eq. (10).

For example, by letting the influence degree of DM1 is $\theta_{1}=8$, and using Eq. (8) his/her normalised expertise is:

$$
\sigma_{k}=\frac{\theta_{k}}{\sum_{l=1}^{K} \theta_{l}}=\sigma_{1}=\frac{\theta_{1}}{\sum_{l=1}^{3} \theta_{l}}=\frac{8}{8+10+7}=0.32
$$

Then the Influenced Closeness Coefficient $I C C_{1,1}^{B}$ for the benefit system for equity E1 according to DM1 is calculated with Eq. (9) as:

$$
I C C_{1,1}^{B}=\sigma_{1} * C C_{1,1}^{B}=0.32 * 0.751=0.2403 \text {, }
$$

and similarly the corresponding Influenced Closeness Coefficient for the cost system $I C C_{1,1}^{C}$ is produces as:

$$
I C C_{1,1}^{C}=\sigma_{1}^{*} C C_{1,1}^{C}=0.32 * 0.457=0.1462 \text {. }
$$

Next, the influenced closeness coefficients have to be normalized prior to matching the coefficients to the linguistic terms in Table 3. Using Eq. (10), $N I C C_{1,1}^{B}$ and $N I C C_{1,1}^{C}$ are calculated as:

$$
N I C C_{1,1}^{B}=\frac{0.2403}{0.2403} \text { and } N I C C_{1,1}^{C}=\frac{0.1462}{0.1659}
$$

Finally, the normalised coefficients are matched to the terms in Table 3:

$$
\begin{aligned}
& N I C C_{1,1}^{B}=1 \cong V G \\
& N I C C_{1,1}^{C}=0.8812 \cong V G
\end{aligned}
$$

Step 7: The antecedent matrices $X_{k}$ for the benefit system are constructed using Eq. (11) for $k=1, \cdots, K$, based on DM $k$ opinions. Each decision maker has a separate benefit antecedent matrix. Similarly, the antecedent matrix $Y_{k}$ is produced for the cost system. Thus the antecedent for the benefit and cost rule bases are generated in this step. For example using Eq. (11), and according to the first decision maker $k=1$, the antecedent matrix $X_{1}$ for the benefit system is:

$$
\begin{aligned}
& \begin{array}{llll}
E_{1} & E_{2} & \cdots & E_{25}
\end{array}
\end{aligned}
$$

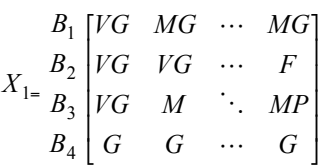

where $B_{i}$ are the four benefit criteria.

Next using Eq. (11) and according to the first decision maker $k=1$, the antecedent matrix $Y_{1}$ for the cost system is:

$$
\begin{aligned}
& \begin{array}{llll}
E_{1} & E_{2} & \cdots & E_{25}
\end{array} \\
& Y_{1}=\begin{array}{l}
C_{1} \\
C_{2}
\end{array}\left[\begin{array}{llll}
G & F & \cdots & F \\
F & G & \cdots & G
\end{array}\right],
\end{aligned}
$$

Step 8: The consequent matrices $\Lambda_{k}$ for the benefit system are constructed using Eq. (12) for $k=1, \cdots, K$, based on the values of $N I C C_{j, k}^{B}$ calculated at Step 5 above and matched to the linguistic terms in Table 3. Similarly, the consequent matrices 
$\Psi_{k}$ are produced for the cost system. Thus the consequent matrix for the benefit and cost rule bases are generated in this step. Having determined the $\operatorname{NICC}_{j}^{B, k}$ and $N I C C_{j}^{C, k}$ coefficients for all decision makers $(k=1, \cdots, K)$, next the benefit consequent matrix $\Lambda_{k}$ and the cost consequent matrix $\Psi_{k}$ are defined using Eq. (12), the consequent matrix $\Lambda_{1}$ is:

$$
\left.\begin{array}{cccc}
E_{1} & E_{2} & \cdots & E_{25} \\
{[V G} & V G & \cdots & G
\end{array}\right] \text { where } B L \text { is the benefit level. }
$$

$\Lambda_{1}=B L\left[\begin{array}{llll}V G & V G & \cdots & G\end{array}\right]$

Then using Eq. (12), the consequent matrix $\Psi_{1}$ is:

$$
\begin{array}{cccc}
E_{1} & E_{2} & \cdots & E_{25} \\
\Psi_{1}=C L\left[\begin{array}{llll}
V G & G & \cdots & G
\end{array}\right]
\end{array} \text { where } C L \text { is the cost level. }
$$

Step 9: Derive rules for each alternative

The rule base of the benefit system for DM1 is constructed using Eq. (13), as follows:

$$
\begin{aligned}
& \text { If } \left.\begin{array}{r|rccc}
E_{1} & E_{2} & \cdots & E_{25} \\
B_{1} & V & M G & \cdots & M G \\
B_{2} & V G & V G & \cdots & F \\
B_{3} & V G & M & \ddots & M P \\
B_{4} & G & G & \cdots & G
\end{array}\right] \\
& \begin{array}{llll}
E_{1} & E_{2} & \cdots & E_{25}
\end{array} \\
& \Lambda_{1}=B L\left[\begin{array}{llll}
V G & V G & \cdots & G
\end{array}\right] ;
\end{aligned}
$$

Then

Rule 1: If $B_{1}$ is $V G$ and $B_{2}$ is $V G$ and $B_{3}$ is $V G$ and $B_{4}$ is $G$ Then the output $B L$ is $V G$,

Rule 2: If $B_{1}$ is $M G$ and $B_{2}$ is $V G$ and $B_{3}$ is $M$ and $B_{4}$ is $G$ Then the output $B L$ is $V G$,

Rule 25: If $B_{1}$ is $M G$ and $B_{2}$ is $F$ and $B_{3}$ is $M P$ and $B_{4}$ is $G$ Then the output $B L$ is $G$.

By analogy, the rule base for the cost system is constructed.

Step 10: The weighted benefit level and weighted cost level are derive by taking average of the aggregate membership value of consequent part of all active rules multiply with weight of system based on number of input for each system. And then multiplying with the influence multiplier based on the average as shown in Eq. (14)

$$
\begin{aligned}
W B L_{1,1}= & {\left[\frac{1}{3}(0.9+0.9+0.9) \times\left(\frac{2}{3}\right)\right] } \\
& \times \frac{1}{3}(1+0.9276+0.9676) \\
& =0.5791
\end{aligned}
$$

and for the cost system

$$
\begin{aligned}
W C L_{1,1}= & {\left[\frac{1}{3}(0.9+0.7+0.9) \times\left(\frac{1}{3}\right)\right] } \\
& \times \frac{1}{3}(0.98823+0.6303+0.8593)=0.2196
\end{aligned}
$$

where $\hat{\mu}_{j, k}^{B}$ and $\hat{\mu}_{j, k}^{C}$ represents aggregate membership value of benefit sub system and cost sub system respectively for each alternative $j=1, \cdots, 25, k$ decision maker.
Step 11: The final score for each alternative $j=1, \ldots, 25$ is derived, by taking average of weighted benefit level and weighted cost level as shown Eq. (15)

$$
\Gamma_{j}=\left(W B L_{j, k}+W C L_{j, k}\right) / 2=\frac{0.5791+0.2196}{2}=0.3993
$$

Step 12: Thus the ranking order of all alternatives can be determined: the better alternatives $j$ have higher values of $\Gamma_{j}$ .The final score and ranking positions for all 25 equities considered in this case study, and based on the proposed MFSTOPSIS method are provided in Table 4

\section{ANALYSIS OF RESULTS}

For the validation of the proposed method, the authors consider established TOPSIS methods such as non-fuzzy, T1, $\mathrm{T} 2$ and $\mathrm{Z}$. All these methods are applied to evaluate the score and final ranking of the equities from the case study in Section $\mathrm{V}$ as well as compared with the performance of MFS-TOPSIS. The actual equity returns based on the traded shares of the 25 companies that are held for a month are used as benchmark ranking. The rankings are compared using the Spearman rho correlation coefficient $\rho$, where $\rho$ measures the strength of association between two ranked variables. This comparison approach is intuitively interpretable and less sensitive to bias due to the effect of outliers [6]. The Spearman's Rho coefficient is evaluated as shown in Eq. (16).

$$
\rho=1-\frac{6 \sum \partial_{i}^{2}}{n^{3}-n},
$$

where $\partial_{i}$ represents the difference between the ranks, and $n$ is the number of considered alternatives. The coefficient $\rho$ takes values between +1 to -1 . Perfect positive relationship of ranks is indicated with $\rho=1$, and $\rho=-1$ indicates perfect negative association of ranks, while $\rho=0$ shows no relationship.

Based on the analysis in Table 5,considering these criteria set i.e. B1, B2, B3, B4, C1 and C2 of financial market described in section III, the novel method Multiple Fuzzy System TOPSIS (NM) outperform the established TOPSIS methods (EM).

\section{CONCLUSION}

This paper introduces a novel TOPSIS method - MFSTOPSIS - extending the capabilities of multiple fuzzy systems within multi-criteria decision-making analysis. MFS-TOPSIS uses fuzzy numbers and incorporates expert knowledge into decision analysis as well as expert degree of experience and influence. At the same time, the approach improves the transparency of the decision making process, particularly in the TOPSIS formulation, by explicitly taking into account all subsystems and interactions among them. MFS-TOPSIS not only provides an effective way to process imperfect information in decision-making practice in a more flexible and intelligent manner but also presents expert knowledge more accurately. The performance of the proposed method is validated using benchmark data and compared against a set of competitive approaches. The results show that the proposed method outperforms the existing non-rule based TOPSIS methods in terms of ranking performance. 
TABLE 4: TOPSIS RANKING PERFORMANCE BASED ON SPEARMAN RHO CORRELATION

\begin{tabular}{|c|c|c|c|c|c|c|c|c|c|c|c|c|c|}
\hline & \multirow{3}{*}{ Actual } & \multirow{2}{*}{\multicolumn{2}{|c|}{ Ranking based on MFS (NM) }} & \multirow{2}{*}{\multicolumn{2}{|c|}{$\begin{array}{c}\text { Non-Fuzzy } \\
\text { Non Fuzzy(EM) }\end{array}$}} & \multicolumn{6}{|c|}{ Fuzzy Non- Rule Based System Approach } & \multirow{2}{*}{\multicolumn{2}{|c|}{$\begin{array}{c}\text { Multiple Fuzzy System } \\
\text { MFS(NM) }\end{array}$}} \\
\hline & & & & & & \multicolumn{2}{|c|}{ T1(EM) } & \multicolumn{2}{|c|}{ T2(EM) } & \multicolumn{2}{|c|}{ Z(EM) } & & \\
\hline & & Final Score & Rank & $\partial_{i}$ & $\partial_{i}^{2}$ & $\partial_{i}$ & $\partial_{i}^{2}$ & $\partial_{i}$ & $\partial_{i}^{2}$ & $\partial_{i}$ & $\partial_{i}^{2}$ & $\partial_{i}$ & $\partial_{i}^{2}$ \\
\hline E1 & 2 & 0.3993 & 4 & 0 & 0 & -2 & 4 & -1 & 1 & -5 & 25 & -2 & 4 \\
\hline E2 & 4 & 0.4002 & 3 & -3 & 9 & 1 & 1 & -1 & 1 & -5 & 25 & 1 & 1 \\
\hline E3 & 1 & 0.4382 & 1 & 0 & 0 & 0 & 0 & 0 & 0 & 0 & 0 & 0 & 0 \\
\hline E4 & 21 & 0.1686 & 20 & 0 & 0 & 1 & 1 & 3 & 9 & 1 & 1 & 1 & 1 \\
\hline E5 & 19 & 0.1396 & 22 & -5 & 25 & -5 & 25 & -4 & 16 & -5 & 25 & -3 & 9 \\
\hline E6 & 11 & 0.3703 & 7 & 5 & 25 & 5 & 25 & 5 & 25 & 3 & 9 & 4 & 16 \\
\hline E7 & 17 & 0.2492 & 12 & 6 & 36 & 5 & 25 & 5 & 25 & 0 & 0 & 5 & 25 \\
\hline E8 & 24 & 0.1743 & 19 & 10 & 100 & 6 & 36 & 7 & 49 & 6 & 36 & 5 & 25 \\
\hline E9 & 23 & 0.1061 & 25 & -2 & 4 & -2 & 4 & -1 & 1 & -2 & 4 & -2 & 4 \\
\hline E10 & 22 & 0.2080 & 17 & 7 & 49 & 6 & 36 & 8 & 64 & 8 & 64 & 5 & 25 \\
\hline E11 & 8 & 0.2323 & 13 & -12 & 144 & -6 & 36 & -7 & 49 & -5 & 25 & -5 & 25 \\
\hline E12 & 13 & 0.2080 & 16 & 1 & 1 & -4 & 16 & -3 & 9 & -3 & 9 & -3 & 9 \\
\hline E13 & 25 & 0.1584 & 21 & 2 & 4 & 3 & 9 & 3 & 9 & 3 & 9 & 4 & 16 \\
\hline E14 & 9 & 0.3321 & 9 & -1 & 1 & 0 & 0 & -1 & 1 & -1 & 1 & 0 & 0 \\
\hline E15 & 3 & 0.3906 & 6 & -5 & 25 & -5 & 25 & -5 & 25 & 0 & 0 & -3 & 9 \\
\hline E16 & 5 & 0.4246 & 2 & 2 & 4 & 3 & 9 & 3 & 9 & 3 & 9 & 3 & 9 \\
\hline E17 & 18 & 0.1317 & 23 & 0 & 0 & -3 & 9 & -3 & 9 & -3 & 9 & -5 & 25 \\
\hline E18 & 12 & 0.1773 & 18 & -7 & 49 & -7 & 49 & -7 & 49 & -7 & 49 & -6 & 36 \\
\hline E19 & 15 & 0.3024 & 11 & 2 & 4 & 4 & 16 & 4 & 16 & 4 & 16 & 4 & 16 \\
\hline E20 & 16 & 0.2139 & 15 & -1 & 1 & 1 & 1 & 3 & 9 & 1 & 1 & 1 & 1 \\
\hline E21 & 7 & 0.3680 & 8 & 3 & 9 & 0 & 0 & 0 & 0 & 3 & 9 & -1 & 1 \\
\hline E22 & 20 & 0.1304 & 24 & -2 & 4 & -3 & 9 & 0 & 0 & -3 & 9 & -4 & 16 \\
\hline E23 & 6 & 0.3957 & 5 & 1 & 1 & 1 & 1 & 2 & 4 & 1 & 1 & 1 & 1 \\
\hline E24 & 14 & 0.3285 & 10 & 5 & 25 & 4 & 16 & 5 & 25 & 8 & 64 & 4 & 16 \\
\hline \multirow[t]{4}{*}{ E25 } & 10 & 0.2250 & 14 & -6 & 36 & -3 & 9 & -15 & 225 & -2 & 4 & -4 & 16 \\
\hline & & & & 0 & 556 & 0 & 362 & 0 & 630 & 0 & 404 & 0 & 306 \\
\hline & \multicolumn{2}{|c|}{ Rho coefficient } & & \multicolumn{2}{|c|}{0.7862} & \multicolumn{2}{|c|}{0.8608} & \multicolumn{2}{|c|}{0.7577} & \multicolumn{2}{|c|}{0.8446} & & 0.8823 \\
\hline & Rank positi & ased performance & & & 4 & & 2 & & 5 & 3 & & & 1 \\
\hline
\end{tabular}

\section{REFERENCES}

[1] Abdollah Hadi-Vencheh and Mahdi Mirjaberi, "Seclusion-Factor Method to Solve Fuzzy-Multiple Criteria Decision-Making Problems," IEEE Trans. Fuzzy Syst., vol. 19, no. 2, 2011.

[2] K. Hwang, C.L.Yoon, Multiple Atribute Decision Making: Methods and Applications. New York: Springer- Verlag, 1981

[3] J.-W. Wang, C.-H. Cheng, and K.-C. Huang, "Fuzzy hierarchical TOPSIS for supplier selection," Appl. Soft Comput., vol. 9, pp. 377-386, 2009.

[4] A. Gegov, N. Petrov, and B. Vatchova, "Advanced modelling of complex processes by rule based networks," 2010 IEEE Int. Conf. Intell. Syst. IS 2010 - Proc., pp. 197-202, 2010.

[5] A. Gegov, Fuzzy Networks for Complex System:A Modular Rule Base Approach. Springer-Verlag Berlin Heidelberg, 2011.

[6] V. G. Hansen, "Detection performance of some nonparametric rank tests and an application to radar," IEEE Trans. Inf. Theory, vol. 16 , no. 3,1970

[7] Tom Clark, "Why Track Actual Costs and Resource Usage On Projects?," ACM, New York, Mar-2008.

[8] Thanh T. Nguyen, L. Gordon-Brown, A. Khosravi, D. Creighton, and Saeid Nahavandi, "Fuzzy Portfolio Allocation Models Through a New Risk Measure and Fuzzy Sharpe Ratio," IEEE Trans. Fuzzy Syst., vol. 23, no. 3, 2015.

[9] R. W. Chen, D. Navin-Chandra, and Fritz B. Prinz, "A CostBenefit Analysis Model of Product Design for Recyclability and its Application," IEEE Trans. Compon. Packaging. Manuf.
Technol., vol. 17, no. 4, 1994.

[10] C.-T. Chen, "Extensions of the TOPSIS for group decisionmaking under fuzzy environment," Fuzzy Sets Syst., vol. 114, no. 1, pp. 1-9, Aug. 2000.

[11] S.-M. Chen and L.-W. Lee, "Fuzzy multiple attributes group decision-making based on the interval type-2 TOPSIS method," Expert Syst. Appl., vol. 37, no. 4, pp. 2790-2798, Apr. 2010.

[12] G. J. Klir, U. St. Clair, and B. Yuan, "Fuzzy set theory: foundations and applications," Apr. 1997.

[13] F. J. J. Santos and H. A. Camargo, "Decision support systems in multicriteria groups: An approach based on fuzzy rules," Int. Conf. Fuzzy Syst., pp. 1-8, Jul. 2010.

[14] A. M. Yaakob and A. Gegov, "TOPSIS Based Selection Alternatives using Interval Type 2- Fuzzy Rule Based System," in IEEE International Conference on Fuzzy Systems, 2015.

[15] J. J. Weygandt, D. E. Kieso, and P. D. \& Kimmel, Accounting principles, 6th Editio. United State of America: John Wiley \& Son, Inc., 2002.

[16] A. Roshayani, U. Laily, and M. A. Siti Maznah, Financial Accounting An Introduction, 2nd Editio. Malaysia: McGrawHill Education, 2007.

[17] F. Tiryaki and M. Ahlatcioglu, "Fuzzy stock selection using a new fuzzy ranking and weighting algorithm," Appl. Math. Comput., vol. 170, no. 1, pp. 144-157, Nov. 2005. 